\title{
Addendum: A Comparison of Angular Values of the Pelvic Limb with Normal and Medial Patellar Luxation Stifles in Chihuahua Dogs Using Radiography and Computed Tomography
}

\author{
Thitaporn Phetkaew ${ }^{1}$ Marissak Kalpravidh ${ }^{1}$ Rampaipat Penchome ${ }^{2} \quad$ Chalika Wangdee $^{1}$ \\ ${ }^{1}$ Department of Veterinary Surgery, Faculty of Veterinary Science,
Chulalongkorn University, Bangkok, Thailand
${ }^{2}$ Imaging Unit, Department of Veterinary Surgery, Faculty of \\ Veterinary Science, Chulalongkorn University, Bangkok, Thailand \\ Address for correspondence Chalika Wangdee, DVM, MSc, PhD, \\ Department of Veterinary Surgery, Faculty of Veterinary Science, \\ Chulalongkorn University, 39 Henri-dunant Road, Bangkok 10330, \\ Thailand (e-mail: c.wangdee@hotmail.com).
}

Vet Comp Orthop Traumatol 2018;31:a1.

Addendum
The authors wish to add the Acknowledgment section in the above article published online in Volume 31, Issue 2 of Veterinary and Comparative Orthopaedics and Traumatology (DOI: 10.3415/VCOT-17-05- 0067). The acknowledgment section has now been added to the article.

\section{Acknowledgments}

This study was supported by the 90th Anniversary of Chulalongkorn University Fund (Ratchadaphiseksomphot Endowment Fund) and Special Task Force for Activating Research (GSTAR 59-007-31-005). We would like to thank the staff of the Department of Veterinary Surgery, Faculty of Veterinary Science, Chulalongkorn University, Thailand. We also acknowledge Achara Tawatsin and Nutthee Am-in for their statistical consultancy and Sanipa Suradhat for proofreading the manuscript. 